\section{Increased mortality in rheumatoid arthritis after a first cardiovascular event}

It is well known that patients with rheumatoid arthritis (RA) have a higher incidence of cardiovascular events than people without RA; however, the risk of death following a cardiovascular event in patients with RA, compared with the general population, has not previously been investigated. Van Doornum and colleagues have now shown that after a first cardiovascular event, patients with RA have a higher mortality rate than people without RA.

The data for this retrospective cohort study were obtained between July 2001 and December 2003 from The Victorian Linked Dataset, which includes information about all episodes of hospital patient care and deaths in Victoria, Australia. According to this dataset, 29,924 patients experienced a first cardiovascular event (myocardial infarction or stroke), of whom 359 had a diagnosis of RA. The 30-day cardiovascular mortality rate was $17.6 \%$ for patients with RA, compared with $10.9 \%$ for patients without RA; the excess mortality in RA patients was almost entirely attributable to an increased number of deaths from myocardial infarction, rather than from stroke. The higher fatality rate in RA patients did not change after adjustment for traditional cardiovascular risk factors, comorbidity and socioeconomic status.

The authors speculate that the increased cardiovascular mortality rate in patients with RA could be caused by the treatments for RA, systemic inflammation, delay in seeking medical attention and in diagnosis, and differences in treatments administered after cardiovascular events; however, the data obtained could not directly address these issues, and further research is required to identify the mechanisms responsible.

Original article Van Doornum S et al. (2006) Increased case fatality rates following a first acute cardiovascular event in patients with rheumatoid arthritis. Arthritis Rheum 54: 2061-2068

\section{Coronary heart disease is often preventable through adherence to a healthy lifestyle}

Certain risk factors for coronary heart disease (CHD), such as hypercholesterolemia, hypertension and smoking, can be modified through lifestyle changes. Chiuve et al. investigated the $\mathrm{CHD}$ burden that may be prevented if individuals adhere to a healthy lifestyle.

The study used data from 42,847 men in the Health Professionals Follow-up Study, who were aged 40-75 years and free from chronic disease at baseline in 1986. Questionnaires every 2-4 years collected data on smoking status, BMI, activity level, diet, and alcohol consumption. For each factor, individuals scored 1 point if they met the criteria for low risk (i.e. not currently smoking, $\mathrm{BMI}<25 \mathrm{~kg} / \mathrm{m}^{2}$, moderate to vigorous activity for $\geq 3.5 \mathrm{~h} /$ week, healthy diet score in top $40 \%$, and moderate alcohol consumption of $5-30 \mathrm{~g} /$ day) and 0 points otherwise. Healthy lifestyle scores were calculated by totaling the number of low-risk lifestyle factors, and therefore ranged from 0 (least healthy) to 5 (most healthy).

Over 16 years of follow-up, 2,183 cases of incident CHD, defined as nonfatal myocardial infarction or fatal CHD, occurred. Healthy lifestyle score was inversely associated with $\mathrm{CHD}$ risk in both the whole population $\left(P_{\text {trend }}<0.0001\right)$ and men already taking lipidlowering or antihypertensive drugs for coronary risk factors $\left(P_{\text {trend }}<0.0001\right)$. The authors estimated that $62 \%$ of CHD cases in this population may have been avoided had all men been at low risk for all five lifestyle factors.

The authors conclude that many cases of $\mathrm{CHD}$ in middle-aged and older men-even in men already taking lipid-lowering or antihypertensive medication-may be avoided if individuals adhere to a healthy lifestyle.

Original article Chiuve SE et al. (2006) Healthy lifestyle factors in the primary prevention of coronary heart disease among men: benefits among users and nonusers of lipidlowering and antihypertensive medications. Circulation 114: 160-167 